Original Article

\title{
Minimal clinically important difference for the Fugl-Meyer assessment of the upper extremity in convalescent stroke patients with moderate to severe hemiparesis
}

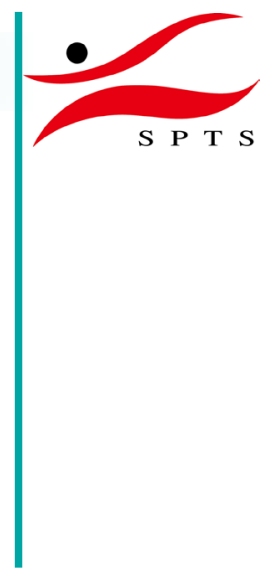

\author{
Shogo Hiragami, OTR, PhD ${ }^{1 *}$, Yu Inoue, RPT, PhD²), Kazuhiro Harada, RPT, PhD ${ }^{3)}$ \\ 1) Faculty of Rehabilitation, Hyogo University of Health Sciences: 1-3-6 Minatojima, Chuo-ku, \\ Kobe-shi, Hyogo 650-8530, Japan \\ 2) Department of Rehabilitation, Kurashiki Heisei Hospital, Japan \\ 3) Department of Physical Therapy, School of Health Sciences and Social Welfare, Kibi International \\ University, Japan
}

\begin{abstract}
Purpose] To estimate the minimal clinically important difference for the Fugl-Meyer assessment of the upper extremity by using anchor-based methods in stroke patients with moderate to severe hemiparesis. [Participants and Methods] Fourteen patients who were hospitalized in a convalescent phase rehabilitation ward were included in this study. Fugl-Meyer assessment of the upper extremity was used to assess the impairment prior to intervention and at follow-up (six weeks later). Participants were asked to evaluate the degree of improvement of paresis of the upper extremity using the global rating of change scale at follow-up. The mean change in Fugl-Meyer assessment scores in the group of patients who answered "a little better, meaningful in daily life" in the global rating of change scale was considered as the minimal clinically important difference. [Results] The mean post-onset period of participants for analysis was 49.4 days. The minimal clinically important difference of the Fugl-Meyer assessment scores were 12.4 (upper extremity), 5.6 (upper arm), and 4.9 (wrist/hand). [Conclusion] A score of 12.4 in the Fugl-Meyer assessment of the upper extremity is likely to be perceived as meaningful in stroke patients with moderate to severe hemiparesis.

Key words: Fugl-Meyer assessment, Minimal clinically important difference, Stroke
\end{abstract}

(This article was submitted Jun. 21, 2019, and was accepted Aug. 7, 2019)

\section{INTRODUCTION}

The most common deficit after stroke is hemiparesis of the upper limb ${ }^{1)}$. The upper-extremity (UE) portion of the FuglMeyer assessment (FMA-UE) is the most frequently used outcome scales to measure poststroke motor recovery of the $\mathrm{UE}^{2,3)}$. Due to severe impact on quality of life, there are a great deal of clinical trials related to hemiparesis of the upper $\operatorname{limb}^{1)}$. This means that there is high demand and utility for clinically valid metrics which can be ascertained using outcome measures such as the FMA-UE. A newer focus within outcome measures is the concept of the minimal clinically important difference (MCID).

MCID is defined as "the smallest difference in score in the domain of interest which patients perceive as beneficial and which would mandate, in the absence of troublesome side effect and excessive cost, a change in the patient's management"4). The use of MCID as an index allows clinicians and researchers to determine whether the change in the scores of outcomes due to intervention indicates meaningful and clinically important improvement for patients ${ }^{5,6)}$. Traditional usage of statistical

*Corresponding author. Shogo Hiragami (E-mail: hiragami@huhs.ac.jp)

(C2019 The Society of Physical Therapy Science. Published by IPEC Inc.

(c) (1) $($ This is an open-access article distributed under the terms of the Creative Commons Attribution Non-Commercial No Derivatives

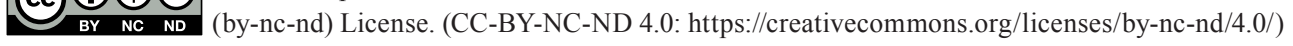


significance (e.g., $\mathrm{p}<0.05$ ) to determine whether an intervention is effective is influenced not only by the extent of change but also the sample size and group variance of the study ${ }^{5,7}$. Thus, if a study observing a stroke group yields a smaller change score than the MCID, this should not be viewed as a significant change to the participants, even if the change in experimental outcome reaches a statistically significant level ${ }^{7}$.

MCID of an instrument can be determined via distribution-based or anchor-based methods ${ }^{5,6)}$. Distribution-based methods estimate MCID values based on the statistical characteristics of scores within a sample ${ }^{5,6)}$. A limitation of the distributionbased method is that derived values do not typically indicate the perception of change of the score by patients. Anchor-based methods estimate MCID values by comparing change scores with the external criteria, usually either the report of a patient or a clinician ${ }^{5,6)}$.

Page et al. reported that in stroke patients with minimal to moderate chronic paresis (baseline FMA-UE scores of $\geq 28$ to $\leq 50$ ), the estimated MCID scores in the FMA-UE were 4.25 (grasping ability), 5.25 (releasing ability), and 7.25 (ability to move arm $)^{8}$. Arya et al. reported that in stroke patients with severe subacute paresis (baseline FMA-UE scores of 14.4), the estimated MCID score of the FMA-UE was 9 to $10^{9}$.

There is often a range in MCID estimates that varies across patient population (e.g., baseline levels of the disability) and clinical study context ${ }^{5,6}$. Thus, a single MCID value may be insufficient across all patient samples. Since levels of UE hemiparesis in stroke patients are diverse ${ }^{10)}$, accumulating evidence of MCID in the FMA-UE at each post-onset period and level of paresis is necessary. Increased knowledge about MCID based on FMA-UE would improve the interpretation of treatment effects in interventional research, thereby improving the clinical management of hemiplegic stroke patients. Therefore, the present study aimed to estimate the MCID of the FMA-UE in stroke patients with moderate to severe hemiparesis in the convalescent phase.

\section{PARTICIPANTS AND METHODS}

In this study, 14 stroke patients who were hospitalized in a convalescent phase rehabilitation ward in 3 hospital in Japan enrolled as participants in a randomized controlled trial $(\mathrm{RCT})^{11)}$ of mirror therapy. Inclusion criteria for the RCT participants were 1) first episode of unilateral stroke with hemiparesis in the prior 1 month, 2) Brunnstrom recovery stage (BRS) ${ }^{12)}$ between I and $\mathrm{V}$ for the hand, 3) no cognitive impairments: mini-mental state examination ${ }^{13)}$ scores $\geq 24$, scores of 0 in consciousness, gaze, visual, language, and extinction and inattention in the National Institutes of Health Stroke Scale ${ }^{14)}$, 4) no muscle spasticity or restriction of range of motion that affecting UE function, and 5) no other disease that influences the affected UE. This study was approved by the Institutional Review Board of Okayama Prefectural University (approval number: 150), and all procedures followed the ethical standards of the Declaration of Helsinki. All participants provided informed consent after receiving both verbal and written information about the study.

We used the FMA-UE to assess recovery from UE impairment. FMA is divided into 5 domains (motor, sensory, balance, range of motion, and pain $)^{2,3)}$. The motor section of FMA-UE evaluates aspects of movement, reflex, coordination, and speed. Each domain contains multiple items, each scored on a 3 -point ordinal scale $(0=$ cannot perform, $1=$ performs partially, $2=$ performs fully). FMA-UE is scored out of 66 , with sub-scores of 36 for the upper arm and 30 for the wrist and hand. In this study, we used all items of the FMA-UE (33 items), 15 items of aspects of movement among items of upper arm (FMA-UA), and 12 items of aspects of movement among items of wrist and hand (FMA-W/H).

The FMA-UE was performed at baseline and at follow-up (6 weeks after baseline). The assessment was conducted by one occupational therapist. We assessed participants UE movement by observing recordings made with a video camera. The physical therapist, who was blinded to the group assignment, scored each item of the FMA-UE based on the video footage. Consistent with other study investigating $\mathrm{MCID}^{4,15)}$, data from all participants were pooled, regardless of group assignment in the trial.

Based on previous studies ${ }^{5,15)}$, the 7-point global rating of change scale (GRC) was used to assess the patient's perception of magnitude of change in the affected UE from baseline. The criterion validity and test-retest reliability of the GRC assessment has been confirmed ${ }^{16}$. At the follow-up assessment, patients were asked by an occupational therapist to answer following question: "How much did your upper extremity paresis change compared to baseline?" using the GRC. Patients responded with a rating based on the following statements: 1-much better, 2-a little better, meaningful in daily life, 3-a little better, not meaningful in daily life, 4-about the same, 5-a little worse, not meaningful in daily life, 6-a little worse, meaningful in daily life, 7-much worse.

In accordance with previous studies ${ }^{5,15}$ ), patients were assigned to the MCID group if their GRC rating was 2 (a little better, meaningful in daily life). The mean changes and rates of change in the score for each FMA-UE, FMA-UA, and FMA-W/H in the MCID group $(\mathrm{GRC}=2)$ were considered as estimated MCID values. All participants which did not answer 1 and 2 on the GRC were classified into the GRC $>2$ group. Differences in the mean change of each FMA-UE, FMA-UA, and FMA-W/H between the groups of $\mathrm{GRC}=2$ and $\mathrm{GRC}>2$ were compared using the Mann-Whitney $\mathrm{U}$ test. Effect size was also calculated as [(mean change in score of each scale in the MCID group) - (mean change in the score of each scale in the GRC $>2$ group)] / the standard deviation of the both groups. Analyses were performed using SPSS version 19 software (IBM, Tokyo, Japan). 


\section{RESULTS}

Of the 14 participants, one was discharged before the follow-up, so we could not conduct the follow-up assessment. In addition, one participant showed perfect FMA-UE scores at follow-up. Excluding these two participants, we analyzed the data of 12 participants. The mean age of participants for analysis was 67.8 years and the mean post-onset period was 49.4 days. At baseline, the BRS was 3.0 (median) for UE and 3.0 (median) for hand, the mean score of the FMA-UE was 27.5 (Table 1, 2).

At follow-up, each FMA-UE, FMA-UA, and FMA-W/H score significantly improved from baseline (Table 2). The distribution of the GRC scales were as follows: $2-$ a little better, meaningful in daily life: $\mathrm{n}=7$ (58.3\%), 3-a little better, not meaningful in daily life: $n=2(16.7 \%)$, and 4 -about the same: $n=3(25.0 \%)$. Five participants who did not answer 1 or 2 on the GRC were classified into the GRC $>2$ group. No participant answered scale 1, 5, 6, or 7 (Table 2). Mean FMA-UE scores and percentage rate of change scores (Table 3) in the MCID group $(\mathrm{n}=7)$ were as follows: FMA-UE: 12.4 (18.8\%), FMA-UA: 5.6 (18.7\%), and FMA-W/H: 4.9 (20.4\%). The scores in the GRC>2 group were as follows: FMA-UE: 5.2 (7.9\%), FMA-UA: 4.0 (13.3\%), and FMA-W/H: $1.0(4.2 \%)$; these were all lower than the MCID group (FMA-UE, p=0.048, ES=0.88; FMA-UA, $\mathrm{p}=0.530, \mathrm{ES}=0.30 ; \mathrm{FMA}-\mathrm{W} / \mathrm{H}, \mathrm{p}=0.106, \mathrm{ES}=0.81)$.

Table 1. Demographic and characteristics of the patients in this study $(\mathrm{n}=12)$

\begin{tabular}{lc}
\hline Gender (Male/Female, $\mathrm{n}$ ) & $6 / 6$ \\
Age (years) & $67.8 \pm 10.5$ \\
Diagnosis, hemorrage/infraction (n) & $4 / 8$ \\
Side of lesions, right/left (n) & $5 / 7$ \\
Affected side, dominant/non-dominant (n) & $7 / 5$ \\
Time since stroke onset (days) & $49.4 \pm 22.2$ \\
MMSE score & $29.4 \pm 1.2$ \\
NIHSS score & $3.3 \pm 3.0$ \\
Brunnstrom stage (UE) (median) & 3.0 \\
Brunnstrom stage (hand) (median) & 3.0 \\
FIM score & $98.0 \pm 17.4$ \\
\hline
\end{tabular}

Values are mean \pm SD or median or n. MMSE: Mini-Mental State Examination; NIHSS: National Institutes of Health Stroke Scale; UE: upper extremity; FIM: Functional independence measure.

Table 2. Mean score of Fugl-Meyer Assessment upper extremity (FMA-UE) at the study baseline and 6 weeks after baseline and frequency of Global rating of change (GRC) (only follow up)

\begin{tabular}{lccc}
\hline \multicolumn{1}{c}{ Measure } & Baseline $(\mathrm{n}=12)$ & Follow up $(\mathrm{n}=12)$ & Test statistics \\
\hline FMA-UE (maximum score=66) & $27.5 \pm 16.5$ & $36.9 \pm 19.3$ & $*$ \\
FMA-UA (maximum score=30) & $13.6 \pm 9.1$ & $18.5 \pm 9.4$ & $*$ \\
FMA-W/H (maximum score=24) & $7.8 \pm 8.4$ & $11.0 \pm 9.2$ & $*$ \\
GRC & & $0(0 \%)$ & \\
2 & & $7(58.3 \%)$ & \\
3 & & $2(16.7 \%)$ & \\
4 & $3(25.0 \%)$ & \\
5 & $0(0 \%)$ & \\
6 & & $0(0 \%)$ & \\
7 & & $0(0 \%)$ & \\
\hline
\end{tabular}

Value are mean $\pm \mathrm{SD}$ or $\mathrm{n} .{ }^{*} \mathrm{p}<0.05$ : Wilcoxon signed-rank test. UA: upper arm; $\mathrm{W} / \mathrm{H}$ : wrist and hand.

Table 3. Estimates of minimal clinically important difference (MICD) for FuglMeyer Assessment upper extremity (FMA-UE)

\begin{tabular}{lcc}
\hline \multicolumn{1}{c}{ Measure } & Raw value $(95 \% \mathrm{CI})$ & Percentage of change score \\
\hline FMA-UE & $12.4 \pm 5.0(7.5-17.4)$ & $18.8 \%$ \\
FMA-UA & $5.6 \pm 2.9(2.7-8.4)$ & $18.7 \%$ \\
FMA-W/H & $4.9 \pm 4.4(0.47-9.24)$ & $20.4 \%$ \\
\hline
\end{tabular}

Value are mean \pm SD. UA: upper arm; W/H: wrist and hand; CI: confidence interval. 


\section{DISCUSSION}

In the present study, we first estimated the MCID of the FMA-UE using patients' perception as external criteria in stroke patients with moderate to severe hemiparesis in the convalescent phase. The mean change between GRC=2 group and GRC $>2$ group was only significantly different for the FMA-UE score. This result may have been influenced by the small sample size of this study. The effect size between the average GRC=2 group and GRC $>2$ group was small-large ${ }^{17)}$. Therefore, classifying participants reporting a GRC score of 2 into the MCID group was considered appropriate. In addition, a study reports that the MCID is beyond 1 SEM (standard error of measurement) ${ }^{7}$. Previous studies have reported 1 SEM of the FMA-UE to be $3.25-3.63^{18,19)}$. In the present study, the MCID was beyond this. Taken together, these results suggest that an MCID based on patients who answered GRC $=2$ is valid.

The MCID score in the present study was higher than that estimated by Page et al., who reported the FMA-UE (overall UE function) score as 5.3 in chronic stroke patients ${ }^{8)}$. Possible reasons for this are firstly, that most recovery from upper extremity paresis occurs in the acute to recovery phase ${ }^{20,21)}$, and secondly, in the chronic phase, during which recovery from paresis is steady, patients may perceive even a little recovery (a small change in the score) as meaningful ${ }^{15}$ ).

Arya et al. reported that in stroke patients with severe subacute paresis, the estimated MCID score of the FMA-UE was 9 to $10^{9}$ ). These patients had similar post-onset periods to our participants, though Arya et al. reported a lower than the MCID score than the scores in the present study ${ }^{9}$. This difference may have been caused by the different levels of paresis at baseline. The degree of recovery from paresis after stroke depends on the level of paresis in the early phase after onset and if the paresis in the early phase after onset is severe, the degree of recovery is likely to be small ${ }^{20,21)}$. Participants in the study by Arya et al. had more severe paresis at baseline than our study (mean score FMA-UE score in Arya et al.: 14.4 compared to 27.5) ${ }^{9}$. This could indicate that paresis at baseline is an important factor for determining MCID. Thus, in the research settings where participants are recovery phase stroke patients with similar paresis levels to those in the present study, an FMA-UE improvement of $>12.4$ points, can be interpreted as a change in score which is perceived as meaningful by the patient.

Another interesting finding of the present study is that the rate of change in the MCID score of FMA-W/H was slightly higher than that of FMA-UA. This suggests that recovery from paresis of the hand and fingers is important for patients to perceive recovery from UE paresis as meaningful.

There are some limitations in this study. First, the sample size of the study was small. Future studies need to be conducted with a larger sample size. Second, using receiver operating characteristics curves ${ }^{8,9)}$ is a useful method for the estimation of MCID, but it was inappropriate in this study due to the small sample size. Third, the degree of paresis and post-onset periods in participants were limited. Since the level of affliction and expression of symptoms of stroke patients are variable, factors such as degree of paresis, time since stroke onset, and whether the affected hand is the dominant hand should be considered when estimating MCID in the future.

In conclusion, our results showed that scores of 12.4 (FMA-UE), 5.6 (FMA-UA), and 4.9 (FMA-W/H) reflected potential MCID scores. These values can be used as a reference by therapists to interpret motor recovery of UE in stroke patients with moderate to severe hemiparesis in the convalescent phase and to develop the appropriate treatment strategy.

\section{Funding}

The authors disclosed receipt of the following financial support for the research, authorship, and/or publication of this article: This work was supported by the JSPS KAKENHI [grant number JP17K12875].

\section{Conflict of interest}

The authors declare no conflict of interest.

\section{REFERENCES}

1) Hatem SM, Saussez G, Della Faille M, et al.: Rehabilitation of motor function after stroke: a multiple systematic review focused on techniques to stimulate upper extremity recovery. Front Hum Neurosci, 2016, 10: 442. [Medline] [CrossRef]

2) Fugl-Meyer AR, Jääskö L, Leyman I, et al.: The post-stroke hemiplegic patient. 1. a method for evaluation of physical performance. Scand J Rehabil Med, 1975, 7: 13-31. [Medline]

3) Gladstone DJ, Danells CJ, Black SE: The fugl-meyer assessment of motor recovery after stroke: a critical review of its measurement properties. Neurorehabil Neural Repair, 2002, 16: 232-240. [Medline] [CrossRef]

4) Jaeschke R, Singer J, Guyatt GH: Measurement of health status. Ascertaining the minimal clinically important difference. Control Clin Trials, 1989, 10: 407-415. [Medline] [CrossRef]

5) Copay AG, Subach BR, Glassman SD, et al.: Understanding the minimum clinically important difference: a review of concepts and methods. Spine J, 2007, 7 : 541-546. [Medline] [CrossRef]

6) Revicki D, Hays RD, Cella D, et al.: Recommended methods for determining responsiveness and minimally important differences for patient-reported outcomes. J Clin Epidemiol, 2008, 61: 102-109. [Medline] [CrossRef]

7) Hsieh YW, Wang CH, Wu SC, et al.: Establishing the minimal clinically important difference of the Barthel Index in stroke patients. Neurorehabil Neural 
Repair, 2007, 21: 233-238. [Medline] [CrossRef]

8) Page SJ, Fulk GD, Boyne P: Clinically important differences for the upper-extremity Fugl-Meyer Scale in people with minimal to moderate impairment due to chronic stroke. Phys Ther, 2012, 92: 791-798. [Medline] [CrossRef]

9) Arya KN, Verma R, Garg RK: Estimating the minimal clinically important difference of an upper extremity recovery measure in subacute stroke patients. Top Stroke Rehabil, 2011, 18: 599-610. [Medline] [CrossRef]

10) Schulz CH, Hersch GI, Foust JL, et al.: Identifying occupational performance barriers of stroke survivors: utilization of a home assessment. Phys Occup Ther Geriatr, 2012, 30: 109-123. [Medline] [CrossRef]

11) Hiragami S, Inoue Y, Sato Y, et al.: The effect of mirror therapy on finger motor dysfunction after stroke. Phys Ther Jpn, 2012, 39: 330-337 (in Japanese).

12) Brunnstrom S: Motor testing procedures in hemiplegia: based on sequential recovery stages. Phys Ther, 1966, 46: 357-375. [Medline] [CrossRef]

13) Folstein MF, Folstein SE, McHugh PR: "Mini-mental state". A practical method for grading the cognitive state of patients for the clinician. J Psychiatr Res, 1975, 12: 189-198. [Medline] [CrossRef]

14) Goldstein LB, Bertels C, Davis JN: Interrater reliability of the NIH stroke scale. Arch Neurol, 1989, 46: 660-662. [Medline] [CrossRef]

15) Lang CE, Edwards DF, Birkenmeier RL, et al.: Estimating minimal clinically important differences of upper-extremity measures early after stroke. Arch Phys Med Rehabil, 2008, 89: 1693-1700. [Medline] [CrossRef]

16) Hiragami S, Inoue Y, Sato Y, et al.: Reliability and validity of the global rating of change scale of upper extremity function in stroke patients (in Japanese). Jpn Occup Ther Res, 2012, 31: 224-232.

17) Cohen J: A power primer. Psychol Bull, 1992, 112: 155-159. [Medline] [CrossRef]

18) Amano S, Umeji A, Uchita A, et al.: Clinimetric properties of the Fugl-Meyer assessment with adapted guidelines for the assessment of arm function in hemiparetic patients after stroke. Top Stroke Rehabil, 2018, 25: 500-508. [Medline] [CrossRef]

19) Kim H, Her J, Ko J, et al.: Reliability, concurrent validity, and responsiveness of the Fugl-Meyer Assessment (FMA) for hemiplegic patients. J Phys Ther Sci, 2012, 24: 893-899. [CrossRef]

20) Duncan PW, Goldstein LB, Horner RD, et al.: Similar motor recovery of upper and lower extremities after stroke. Stroke, 1994, 25: 1181-1188. [Medline] [CrossRef]

21) Duncan PW, Goldstein LB, Matchar D, et al.: Measurement of motor recovery after stroke. Outcome assessment and sample size requirements. Stroke, 1992, 23: 1084-1089. [Medline] [CrossRef] 